\title{
COMMENTS ON THE INFLUENCE OF SOLAR ACTIVITY ON P-MODE OSCILLATION SPECTRA
}

\author{
L. GIZON \\ W.W. Hansen Experimental Physics Laboratory, \\ Stanford University, Stanford CA 94305, U.S.A.
}

\section{Spectral signature of a persistent patch of activity}

The systematic p-mode frequency changes which are observed through the solar cycle are believed to be associated with near-surface perturbations confined to the magnetically active latitudes. In this paper, we study the perturbation arising from the presence of a large "active region", corresponding to a localized structural change in a thin region close to the photosphere. We shall ignore the difficult question of the magnitude of the effect, and simply consider some geometric and observational implications for low-degree modes.

The technique (Gizon, 1995) consists of calculating the eigenstates in a frame in which both the active region and the rotation can be assumed to be steady perturbations (see also e.g. Gough and Taylor, 1984). Figure 1 schematically displays the eigenfrequencies of an $l=2$ multiplet. Rotation was presumed to be known (Kosovichev, Schou, Scherrer, et al., 1997). In a frame co-rotating with the active region, the $(2 l+1)$-fold azimuthal degeneracy is completly lifted. Because nonuniform splitting due to differential rotation is small, and because the spatial extent of the active

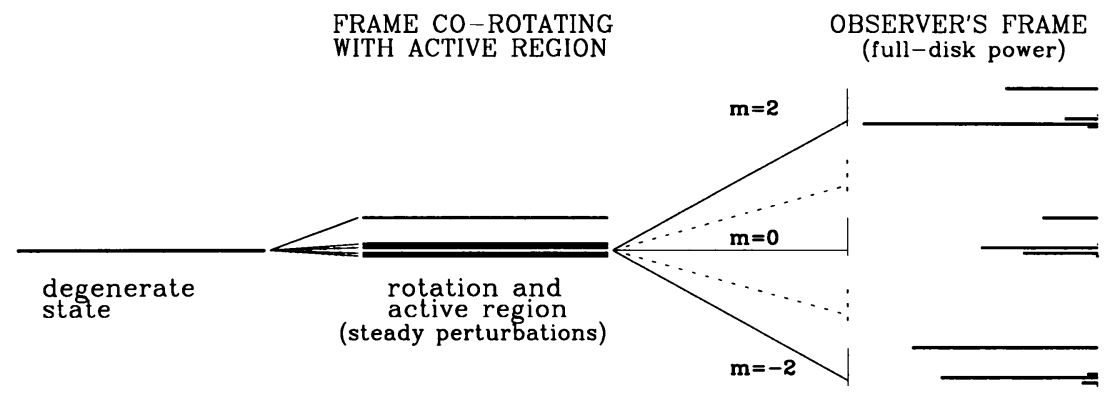

Figure 1. Energy diagram for a quadrupole mode in a frame co-rotating with the active region. Also shown is the full-disk power spectrum seen by an observer on Earth or by SOHO. In this diagram, the assumed perturbation from the active centre, lying at latitude $5^{\circ}$, produces a mean frequency shift of the whole multiplet of $80 \mathrm{nHz}$. 

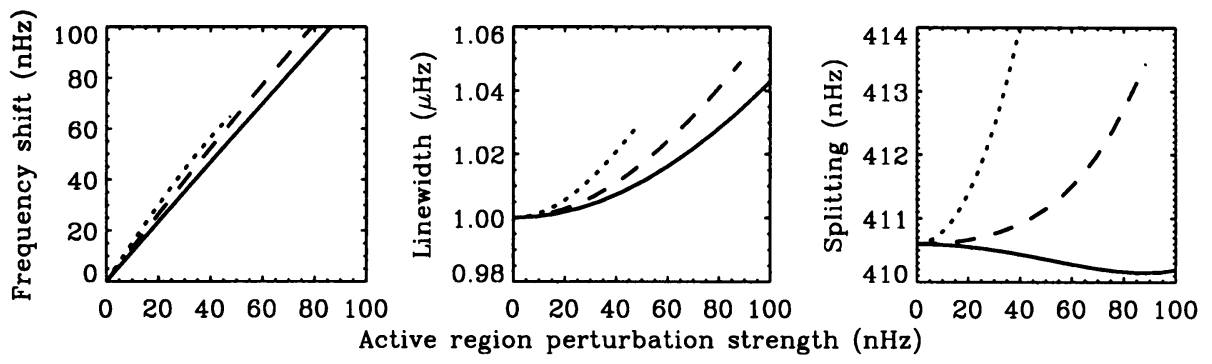

Figure 2. Estimates of the frequency shift, linewidth and rotational splitting of the $(l=2$, $n=20$ ) multiplet, versus active region perturbation strength (expressed in terms of the power-weighted average of the $(l+1)(2 l+1)$ individual frequency shifts). Each individual peak composing the multiplet is assumed to have a linewidth of $1 \mu \mathrm{Hz}$. The solid, dashed and dotted lines refer to the active-region latitudes $5^{\circ}, 20^{\circ}$ and $35^{\circ}$ respectively.

region is also small, only one of the eigenfrequencies (in co-rotating frame) is significantly affected. The fine separation between the other $2 l$ components is essentially due to differential rotation. For an observer orbiting about the Sun's rotation axis at the Earth's orbital angular velocity, the full-disk power spectrum appears to have $(l+1)(2 l+1)$ peaks, most of which cannot be resolved (Figure 1).

\section{Some observational consequences}

We may generate the synthetic profile of an $l=2$ multiplet by adding the 15 individual profiles, represented by Lorentz curves of equal linewiths. Usually, observers model the expectation of the power spectrum by the sum of three Lorentz functions of unknown amplitudes. Figure 2 shows the errors in the determination of the parameters by use of this approximate model. Estimates of the parameters are obtained by a leastsquares technique, where each point is assigned a weight inversely proportional to its value. We see that the centroid frequency and the linewidth are likely to be slightly overestimated. The error in the rotational splitting frequency depends on the latitude of the active region. Of course, the magnitudes of these errors ultimately depend on the physics, and also on the fitting procedure which is used.

Dziembowski and Goode (1997) suggested that seismic information about the solar core should be purged from the Sun's magnetic activity. The latitudinal structure of the near-surface perturbation has to be taken into account in this process. I suggest that longitudinal variations could have some importance as well. A realistic study would have to consider the fact that solar activity is a transient phenomenon.

I thank C.J. Durrant, D.J. Galloway, D.O. Gough and P.R. Wilson for discussions.

\section{References}

Dziembowski, W.A. and Goode, P.R.: 1997, Astron. Astrophys. 317, 919.

Gizon, L.: 1995, in Vincent, R.A., et al. (eds.), Conf. on Solar and Terrestrial Physics STEP'95, University of Adelaide, Adelaide, pp. 173-176.

Gough, D.O. and Taylor, P.P.: 1984, Mem. S.A.It. 55, 215.

Kosovichev, A.G., Schou, J., Scherrer, P.H., Bogart, R.S., Bush, R., Hoeksema, J.T., et al.: 1997, Solar Phys. 170, 43. 